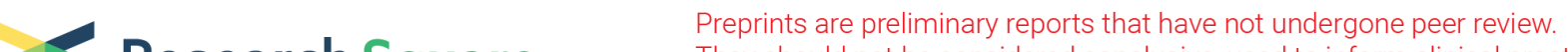 Research Square They should not be considered conclusive, used to inform clinical practice, or referenced by the media as validated information.
}

\section{The Agonist of JWA Gene JAC1 Suppresses Proliferation of Breast Cancer Through the JWA/p38/SMURF1/HER2 Signaling}

\section{Yanlin Ren}

School of Public Health,Nanjing Medical University

\section{Dongyin Chen}

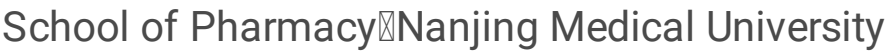

Junjie Chen

School of Public Health,Nanjing Medical University

Zurong Zhai

School of Public Health,Nanjing Medical University

Aiping Li

School of Public Health,Nanjing Medical University

\section{Yan Liang}

The first Affiliated Hospital of Nanjing Medical University,Nanjing

Jianwei Zhou ( $\boldsymbol{\sigma}$ jwzhou@njmu.edu.cn )

School of Public Health, Nanjing Medical University

\section{Research}

Keywords: JWA agonist, Breast cancer, HER2, E3 ubiquitin ligase, SMURF1

Posted Date: June 26th, 2020

DOl: https://doi.org/10.21203/rs.3.rs-37842/v1

License: (c) (i) This work is licensed under a Creative Commons Attribution 4.0 International License. Read Full License 


\section{Abstract \\ Background}

The overexpression of HER2 is associated with malignant proliferation and invasiveness in breast cancer. Although HER2-targeting drugs have been clinically applied for cancer treatment, none of them could reduce overexpressed HER2. In this study, we reported that JAC1 could suppress proliferation of breast cancer cells via degrading HER2.

\section{Methods}

JWA-HER2 association was analyzed by IHC in 90 paired cases of breast cancer and adjacent noncancerous tissues. Regulatory effect of JAC1, the agonist of JWA gene, on HER2-positive breast cancer cells was studied using colony formation assay. The effect of JAC1 on the localization of HER2 was detected by immunofluorescence microscopy assay. Western blotting, RT-PCR and immunoprecipitation assay were utilized to investigate the mechanisms of JWA on regulating HER2. Finally, xenograft mouse models were established in nude mice using BT474 cells to confirm the effect of JAC1 in vivo.

\section{Results}

JAC1, a small molecule agonist of JWA gene, dose-dependently suppressed proliferation in HER2-positive breast cancer in vitro and in vivo through degrading HER2. The mechanistic evidences showed that JAC1 increased the ubiquitination of HER2 at the K716 through the E3 ubiquitin ligase SMURF1. Furthermore, SMURF1 was activated due to reduced expression of NEDD4, an E3 ubiquitin ligase for SMURF1 through the JWA-p38-GATA-1-NEDD4 axis.

\section{Conclusions}

JAC1 suppresses the proliferation in HER2-positive breast cancer through the JWA/p38/GATA1/NEDD4/SMURF1/HER2 signaling. JAC1 may serve as a novel therapeutic agent to breast cancer.

\section{Background}

Despite important advances in cancer research, BC poses a huge threat on females health, with the highest morbidity and mortality among female cancers worldwide[1]. Therefore, BC has become a major problem in public health management and has been well concerned in biomedical research[2, 3]. BC is classified into four major molecular subtypes, including luminal A, luminal B, HER2-positive and normal breast-like cancer[4]. Human epidermal growth factor receptor (HER2/ErbB2), a member of the epidermal growth factor receptor (EGFR) family, is negatively or weakly expressed in normal cells. However, it is abundantly expressed in some cases of BC and associated with proliferative and invasive capacities of 
cancer cells[5]. In the last two decades, BC patients have been impressively benefited from monoclonal antibodies (e.g. trastuzumab) and tyrosine kinase inhibitors (e.g. Lapatinib) directly against HER2[6-8]. Unfortunately, side effects (e.g. bone marrow suppression, cardiotoxicity and hepatotoxicity) and the resistance of anti-HER2 therapies significantly restrict the therapeutic efficacy[9-11]. To avoid the side effects of completely blocking HER2, novel drugs or therapies that are capable of down-regulating overexpressed HER2 are expected.

The JWA gene, also known as ARL6IP5, is a multifunctional and cytoskeleton binding protein. The functions of JWA gene are involved in regulating oxidative stress, DNA repair, cell apoptosis and differentiation[12, 13]. Highly expressed JWA is a favorable prognostic indicator for gastric and breast cancer[14]. Patients with lower expression of JWA in tumor tissues usually suffer worse survival than those with higher expression[15, 16]. JWA negatively regulates HER2 expression and inhibits cell proliferation and migration in human GC cells through transcription and ubiquitination related mechanisms $[17,18]$. However, the role of JWA in regulating HER2 expression and its underlying mechanism in $\mathrm{BC}$ have not been determined.

In the present study, we investigated the potential role of JAC1, the agonist of JWA gene on HER2 positive $\mathrm{BC}$ cells in vitro and in vivo models. We demonstrated at first time that JAC1 downregulated the overexpressed HER2 in BC cells and suppressed the proliferative potential via activation of JWA gene. JAC1 triggered a two-stage ubiquitination modification on SMUF1 and HER2 driven by p38. Therefore, our data may provide a novel therapeutic target for HER2-positive BC.

\section{Materials And Methods}

\section{Cell culture}

BT474 and SKBR3 human BC cells were obtained from the American Type Culture Collection (ATCC). The cells were cultured in DMEM supplemented with $20 \%$ and $10 \%$ fetal bovine serum (FBS), respectively at $37{ }^{\circ} \mathrm{C}$ in a humidified atmosphere containing $5 \% \mathrm{CO}_{2} .100$ units/ml penicillin and streptomycin (Cellgro, Hemdon, VA) were supplemented in the culture medium.

\section{Western blotting}

Western blot assays were performed based on the previously reported protocols[19]. The following antibodies were used: Anti- $\beta$-actin; anti-a-tubulin; anti-GAPDH; anti-HA (1;1000, Beyotime, Jiangsu, China); anti-HER2; anti-HER3; anti-p-p38(Thr180/Thr182); anti-Ub (1:1000, CST, USA); anti-JWA (1:100, Laboratory-made); anti-GATA-1; anti-NEDD4; anti-CBL; anti-SMURF1; anti-HER1; and anti-HER4 (1:1000, proteintech, China).

\section{Colony formation assay}

BT474 and SKBR3 cells were treated by 1 or $10 \mu \mathrm{M}$ JAC1 for 10-14 days and not harvested until the formation of visible colonies. BT474 and SKBR3 cell colonies were washed twice with PBS, fixed with 
methanol and stained with crystal violet. Only the colonies with > 50 cells were counted.

\section{Immunofluorescence microscopy}

BT474 and SKBR3 cells were induced with JAC1 or vehicle (DMSO) for $24 \mathrm{~h}$. Subsequently, the cells were fixed with methanol for 30 min, washed with PBST and $10 \%$ normal goat serum for $1 \mathrm{~h}$ aiming to block non-specific signals. The cells were incubated with anti-JWA antibody (1:200) and anti-HER2 (1:250) overnight at $4{ }^{\circ} \mathrm{C}$. After washing with PBST, the FITC goat anti-mouse IgG and CY3 goat anti-rabbit IgG (1:100, Beyotime, Jiangsu, China) were used to incubate with cells for $2 \mathrm{~h}$. After washing three times with PBST, the cells were stained with DAPI (Beyotime, Jiangsu, China) for $20 \mathrm{~min}$. The confocal images of the cells were captured using Zeiss AIM software on a Zeiss LSM 700 confocal microscope system (Carl Zeiss Jena, Oberkochen, Germany).

\section{CRISPR/Cas9 system}

The CRISPR/Cas9 system was used to disrupt the expression of the JWA gene, as described elsewhere[20]. In brief, an sgRNA sequence was selected using an Optimized CRISPR Design (http://crispr.mit.edu/). The sgRNA sequence for JWA was 5'-CCGCGTAGTGAGCAACCTGCTCT-3'. For sequence analysis of the JWA gene, the following primer set was used: 5'-CACTCAGTCCCTTGTTCTGTC3' (forward) and 5'-GCACCAAGGGCTGAGACTT-3' (forward).

\section{Quantitative real-time RT-PCR assay}

Total RNA was extracted from harvested cells using the Trizol reagent (Gibco, USA) according to the manufacturer's instructions. Approximately $500 \mathrm{ng}$ of RNA was reversely transcribed with Hiscript Q RT SuperMix for qPCR (Vazyme, Jiangsu, China). The primers used in this work are described in Additional files 1: Experimental Procedures (Word).

\section{Plasmids and siRNA transfection}

The construction of Flag-JWA and Ub plasmids were introduced in our previous study[21]. The HA-HER2 plasmid and corresponding mutants were subcloned into a pcDNA3.1 vector (Generay, Shanghai, China) using HindIII/Xhol sites. The siRNA sequences (5'-CGAGCUAUUUCCUUAUCUC-3') were used for the JWA; the siRNA sequences (5'-GCAGAACAGGCUGAGGAAUTT-3') were used for the NEDD4; the siRNA sequences (5'-CCAGGGAGUGGCUUUACUUTT-3') were used for the SMURF1 and a nonsense control siRNA were synthesized by Ribobio (Guangzhou, China). The plasmids and siRNA were transfected into cells with Lipofectamine 3000 according to manufacturer's instructions (Invitrogen, Grand Island, NY, USA).

\section{Immunoprecipitation assay}

This procedure was conducted using protein A/G Plus-Agarose (Santa Cruz, USA) according to the manufacturer's instructions. Briefly, cells were treated as indicated treatments and were lysed in TNE buffer (50 mM Tris-HCl, $150 \mathrm{mM} \mathrm{NaCl}, 1 \%$ NP40) containing protease inhibitors (Sigma Aldrich, CA, USA).

\section{Ubiquitination assay}


BT474 and SKBR3 cells transiently transfected with $\mathrm{Ub}$ for $72 \mathrm{~h}$ were incubated with $10 \mu \mathrm{M} \mathrm{JAC} 1$ for $24 \mathrm{~h}$, followed by MG132 $(10 \mu \mathrm{M})$ induction for another $6 \mathrm{~h}$. The cells were harvested for extracting proteins. Protein samples were incubated with anti-HER2 antibody $(1: 200, \mathrm{CST}, \mathrm{USA})$ at $4{ }^{\circ} \mathrm{C}$ for $1 \mathrm{~h}$, and then Protein A/G Plus-Agarose overnight. Pre-cooled IP buffer was used to wash cells for four times. After centrifugation at $4{ }^{\circ} \mathrm{C}, 1000 \cdot \mathrm{g}$ for $5 \mathrm{~min}$, the obtained IP product was examined by western blot.

\section{Tissue microarray (TMA) and immunohistochemical (IHC) staining}

The details of TMA and IHC staining are described in Additional files 1: Experimental Procedures (Word).

\section{Tumor xenografts}

This study was approved by the Ethics Committee of Nanjing Medical University and the Approval Number was IACUC-1811067. The details of tumor xenografts are described in Additional files 1: Experimental Procedures (Word).

\section{Statistical analysis}

Statistical analysis was performed using SPSS software (version 23.0, Inc., Chicago, IL) and GraphPad Prism 6 software (GraphPad Software, Inc., La Jolla, CA, USA). The differences between two independent groups were analyzed by Student's $t$ test (unpaired, two-tailed). For each significantly ectopically expressed molecule, Kaplan-Meier overall survival and relapse-free survival analyses were performed with a log-rank test for comparing differences between curves. $P<0.05$ was considered statistically significant (* $P<0.05 ;$;* $P<0.01 ;$ *** $P<0.001$ ).

\section{Results}

JWA negatively regulates HER2 expression and cell proliferation in BC.

To identify the potential involvement of JWA in HER2-positive BC, firstly, we interrogated the TCGA database. Compared with normal breast epithelium, JWA was significantly downregulated in breast tumors ( $n=1218$; Fig. 1a). The overall survival (OS) was longer in cases with higher expression of JWA in tumor tissues than those with lower expression. Meanwhile, the OS was shorter in cases with higher expression of HER2 than those with lower expression of HER2 $(n=1402$; Fig. $1 \mathrm{~b}-\mathrm{c})$. To confirm this finding, we analyzed survival data from another database (GSE88770 and GES42568, $n=221$ ). The data consistently showed that low expressed JWA or high expressed HER2 was unfavorable to the prognosis in BC. In contrast, BC cases with high level of JWA and low level of HER2 had a better outcome (log-rank test, $* P<0.05 ; * \star P<0.01$; Fig. 1d). We further verified protein expressions of JWA and HER2 in human BC tissue microarray (TMA). As predicted, significantly downregulated JWA was observed in cancer samples with HER2 overexpression (Fig. 1e). As shown in Additional files 2: Figure. S1a-b, 32/44 cases showed low expression of JWA in HER2-positive ones. In contrast, 27/46 cases displayed high expression of JWA in HER2-negative ones $(n=90 ; P<0.005)$. 
To determine the potential influence of JWA on HER2 expression, we constructed Flag-JWA, si-JWA and their controls. They were transfected into human BC BT474 cells, respectively. As shown in Fig. 1f, HER2 was down-regulated in cells transfected with Flag-JWA, whereas an up-regulation of HER2 was observed in cells transfected with si-JWA. Therefore, HER2 expression was negatively regulated by JWA in BC cells. To determine the regulatory effect of JWA on proliferation of BC cells, we conducted colony formation assay. An obvious inhibition of proliferation was observed in BT474 cells transfected with Flag-JWA. On the contrary, an increased proliferation in BT474 cells transfected with si-JWA was determined $(P<0.005$; Fig. 1g). Confocal imaging assay showed that the expression of HER2 was negatively regulated by JWA in BT474 cells (Fig. 1h).

\section{JAC1 down-regulates expression of HER2 and inhibits cell proliferation in BC.}

To find the potent agonist of JWA gene in human normal cells, we constructed JWA promoter-containing reporter gene stably transfected HBE cells. A series of high-throughput screening assays on 40,000 compounds were conducted in national compound library (Shanghai, China). Finally, there are six proper candidates that could activate the expression of JWA (JAC1 JAC6) (Fig. 2a). As shown in Additional files 3: Figure. S2a-b, JAC1 was more effective in inhibiting cell proliferation than the other 5 candidates. In addition, JAC1 induction displayed better capacities in activating JWA and down-regulating HER2 in BT474 cells than other 5 candidates (Additional files 3: Figure. S2c). The chemical structure formula of JAC1 was shown in Additional files 4: Figure. S3a. JAC1 up-regulated JWA and down-regulated HER2 expressions at protein levels in both BT474 and SKBR3 cells in a dose-dependent manner (Fig. 2b). The $\mathrm{IC}_{50}$ values of JAC1 are between $25 \mu \mathrm{M}$ and $50 \mu \mathrm{M}$ in BT474 and SKBR3 cells (Additional files 4: Figure. S3b-c).

To confirm if JWA was responsible for JAC1-induced regulation on HER2 in cancer cells, we constructed JWA knockout (KO) SKBR3 human BC cells by CRISPR/Cas9 technique. DNA sequencing results showed that the 313th base of JWA was missing A base and randomly inserted CT bases (Additional files 4: Figure. S3d). An off-target assay of JAC1 on HER2 expression was carried out in JWA KO SKBR3 cells. As indicated in Figure. 2C, JAC1 activated JWA, but suppressed HER2 expression significantly in JWA wildtype (WT) SKBR3 cells. In JWA KO SKBR3 cells, however, JAC1 had no regulatory effects on HER2 expression. Colony formation assay showed that JAC1 dose-dependently inhibited proliferation in both BT474 and SKBR3 cells (Fig. 2d-e). Confocal imagining showed a dose-dependent reduction of HER2 expression, but increase of JWA expression in both BT474 and SKBR3 cells after treatment with JAC1 for 24 h (Fig. 2f).

\section{JAC1 down-regulates expression of HER2 via the ubiquitin-proteasome pathway.}

To determine how JAC1 down-regulates HER2 in BC cells, we first examined mRNA level of HER2 by RTPCR after JAC1 treatment. The data showed that JAC1 increased JWA level, but had non-effect on HER2 at mRNA level in both BT474 and SKBR3 cells (Additional files 5: Figure. S4a-b). At protein level, HER2 degradation was obviously accelerated by JAC1 treatment in both BT474 and SKBR3 cells following CHX 
exposure at the indicated time points (Fig. 3a and Additional files 5: Figure. S4c). We further demonstrated that ubiquitinated HER2 obviously up-regulated in both BT474 and SKBR3 cells after JAC1 exposure (Fig. 3b-c). Potential candidates of E3 ubiquitination ligase to HER2 were subsequently searched in the UbiBrowser (ubibrowser.ncpsb.org/)[22]. In this network view, the central node and the surrounding nodes were the queried substrate ERBB2 (HER2) and the predicted corresponding E3 ubiquitination ligases, respectively. The predicted data have shown that NEDD4, CBL and SMURF1 may exert vital role in ubiquitination degradation of HER2 (Fig. 3d). As a result, among the three candidates, SMURF1 expression was up-regulated but NEDD 4 was down-regulated dose-dependently by JAC1 treatment in both BT474 and SKBR3 cells (Fig. 3e and Additional files 5: Figure. S4d). Similar results were obtained after transfection of either Flag-JWA or si-JWA in SKBR3 cells (Additional files 5: Figure. S4e). These data suggested that SMURF1 may be the candidate E3 ubiquitin ligase for HER2. We then conducted co-immunoprecipitation experiments to elucidate the potential interaction between SMURF1 and HER2. As predicted, there was an interaction between HER2 and SMURF1 in BT474 and SKBR3 cells (Fig. $3 f$ and Additional files 5: Figure. S4f). The interactions between HER2 and NEDD4, HER2 and CBL were also tested. Unfortunately, we obtained negative results (Additional files 5: Figure. S4g-j). In addition, the inhibition of SMURF1 expression by si-SMURF1 can reverse the degradation of HER2 by JAC1 (Fig. 3g).

\section{K716 of HER2 is the ubiquitination target by SMURF1.}

To identify the potential target amino acids in HER2 by SMURF1 ubiquitination, we searched online database (http://www.phosphosite.org/). As shown in Fig. 4a, there were 10 candidate ubiquitination sites (lysine, $k$ ) in HER2 protein amino acid sequence. We then constructed HER2 mutants for all or separated of these 10 amino acids in HER2 sequence and replaced lysine (K) by arginine (R), respectively. Next, wild-type or mutant-type HER2 plasmids were transfected into SKBR3 cells respectively, followed by JAC1 and CHX treatment. As a result, only HER2 (K716R) and HER2 (ALL) (all 10 sites mutant) were resistant to CHX-induced reduction of HER2 (Fig. 4b). To confirm this finding, HER2 (K716R), HER2 (WT) and HER2 (ALL) mutant were transfected into BT474 cells, respectively, followed by treatment with JAC1 and $\mathrm{CHX}$. Similar results were yielded in BT474 cells that JAC1 only accelerated degradation of HER2 (WT), rather than HER2 (K716R) and HER2 (mutant ALL) (Fig. 4c). Then, the ubiquitination of HA-HER2 was attenuated by MG132 treatment in HER2 (K716R) SKBR3 cells compared to those with HER2 (WT) cells (Fig. 4d). Colony formation numbers were increased in HER2 (WT) and HER2 (K716R) BT474 cells compared to control cells $(P<0.001)$. JAC1 $(10 \mu \mathrm{M})$ treatment obviously suppressed cell proliferation in both HER2 (WT) and control BT474 cells, but it had no effect on HER2 (K716R) BT474 cells (Fig. 4e-f). Thus, the effect of JAC1 on BC cell proliferation was dependent on the presence of amino acid K716 in HER2.

\section{JAC1 modulates SMURF1 through JWA-p38-GATA-1-NEDD4 axis}


Given JAC1 as potent activator of SMURF1, we desired to identify how JAC1 up-regulates SMURF1. As predicted online, NEDD4 was the most crucial E3 ubiquitin ligase of SMURF1 (Additional files 6: Figure. S5a) since it was dose-dependently down-regulated by JAC1 treatment (Fig. 3e and Additional files 5: Figure. S4d). The expression patterns of SMURF1 and NEDD4 were quite oppositely after JAC1 exposure. In addition, co-IP experiments manifested the interaction between SMURF1 and NEDD4 (Fig. 5a-b). The interaction between SMURF1 and NEDD4L was also tested. Unfortunately, we obtained a negative result (Additional files 6: Figure. S5b). Transfected with si-NEDD4, the expression of NEDD4 was decreased and the expression of SMURF1 was significantly increased in SKBR3 cells. However, the expression of SMURF1 was decreased but the expression of NEDD4 was not affected after SKBR3 cells were transfected with si-SMURF1. Knocking down both NEDD4 and SMURF1, the increased SMURF1 was inhibited. These results indicated that NEDD4 was an upstream regulatory molecule of SMURF1 and was involved in negative regulation of the expression of SMURF1 (Fig. 5c).

To elucidate how JAC1 down-regulates NEDD4 expression, we determined mRNA level of NEDD4 by RTPCR. The data showed that JAC1 dose-dependently down-regulated mRNA expression of NEDD4 (Fig. 5d and Additional files 6: Figure. S5c). Further, online prediction (UCSC and ALGGEN-PROMO) indicated that several transcription factors (GATA-1, ER-alpha, STAT4, STAT1-beta, GR-alpha and TFIID) may contribute to the JAC1-mediated NEDD4 down-regulation (Additional files 6: Figure. S5d). Non-regulation between ER and HER2 has been already identified. RT-PCR results showed that JAC1 down-regulated mRNA expression of GATA-1, but had no effect on the mRNA levels of STAT4, STAT1-beta, GR-alpha and TFIID (Fig. 5e). Western Blot results showed that JAC1 treatment induced a dose-dependent increase of JWA and reduction of GATA-1 level (Fig. 5f). Recent studies have revealed that JWA inhibits cell migration by activating the MAPK signaling pathway[13,23], and TNF-a represses GATA-1 through the activation of p38 MAPK[24]. Here we identified that expression of GATA-1 was negatively regulated by JAC1-mediated activation of p38 MAPK (Fig. 5g). To confirm this speculation, p38 inhibitor SB203580 was used to block p38. Data showed that the expression of p-p38 induced by JAC1 was mostly prevented by SB203580. Furthermore, the inhibitory effects of JAC1 on the expressions of GATA-1, NEDD4 and HER2 were mostly reversed by SB203580 treatment; meanwhile, the activation effect of JAC1 on the expression of SMURF1 was reversed by SB203580 treatment in SKBR3 cells (Fig. 5h). To further confirm the involvement of this pathway in guiding JAC1-regulated proliferation in $\mathrm{BC}$, colony formation assay was conducted. The data showed that the suppressive role of JAC1 in proliferation of SKBR3 cells was partly reversed by SB203580 (Fig. 5i). Taken together, we have revealed that JAC1 inhibited proliferation in BC cells by suppression of HER2.

\section{JAC1 suppresses tumor growth in $\mathrm{BC}$ xenografted mice}

To evaluate the translational significance, an in vivo BC xenograft model by implanting BT474 cells and JAC1 experimental therapy model were conducted in mice. As shown in Fig. 6a, JAC1 dose-dependently inhibited the growth of xenografted tumor in mice. The similar trend of tumor weight/body weight was shown in Fig. 6b. The tumor inhibition rate by JAC1 was $31.22 \%$ and $46.21 \%$ in $50 \mathrm{mg} / \mathrm{kg}$ and $100 \mathrm{mg} / \mathrm{kg}$ groups, respectively (Fig. 6c). In addition, compared to the mock group, the solvent showed no significant 
effect on tumor growth (Fig. 6a-c and Additional files 7: Figure. S6a-b). To confirm the in vivo activation of p38 signaling in JAC1-induced tumor growth inhibition, relevant biomarkers were determined in isolated BC tissues. As shown in Fig. 6d, expression level of HER2 was dose-dependently reduced by JAC1 treatment. Importantly, the mechanistic biomarkers expressions, including JWA, p-p38, GATA-1, NEDD4 and SMURF1 were correspondingly changed in tumor tissues. H\&E staining revealed that JAC1 treatment did not induce obvious injuries on organs, including lung, liver, spleen, kidney and myocardium (Additional files 7: Figure. S6c). The serum biochemical parameters indicated a favorable improvement after JAC1 treatment, including antioxidant (SOD), liver function (ALT/AST), myocardial enzymes (CKMB/CK) and lipid metabolisms (TG) (Table 1). Taken together, the in vivo results demonstrated that JAC1 inhibits breast tumor growth in xenografted mice in a dose-dependent manner, which was consistent to the findings obtained from in vitro models (Fig. 6e). In conclusion, JAC1, the agonist of JWA gene, leaded to phosphorylation of p38 and subsequently down-regulated the GATA1, the transcription factor of E3 ubiquitin ligase NEDD4. Silenced NEDD4 resulted in an overexpression of SMURF1, a specific E3 ubiquitin ligase for HER2 (K716). With the degradation of HER2, the tumor growth was inhibited in HER2-positive BC. In particular, JAC1 has a protective effect on major organs and lipid metabolism in mice. 
Table 1

The serum biochemical indexes (21 parameters) in control and $100 \mathrm{mg} / \mathrm{kg}$ JAC1 treated mice.

\begin{tabular}{|c|c|c|c|}
\hline \multirow[t]{2}{*}{ Analysis } & \multicolumn{3}{|l|}{ Groups } \\
\hline & Mock & 0 mg/kg JAC1 & 100 mg/kg JAC1 \\
\hline [ALT] & $29.33 \pm 2.52$ & $30 \pm 4.24$ & $23.75 \pm 2.22 \star$ \\
\hline [AST] & $134.75 \pm 18.46$ & $136 \pm 16.19$ & $97 \pm 19.61^{\star}$ \\
\hline [TP] & $39.03 \pm 3.44$ & $42.24 \pm 5.07$ & $42.16 \pm 5.21$ \\
\hline [ALB] & $26.23 \pm 0.81$ & $27.35 \pm 3.48$ & 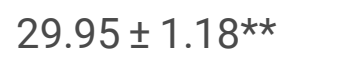 \\
\hline [TBIL] & $0.47 \pm 0.15$ & $0.43 \pm 0.15$ & $0.58 \pm 0.1$ \\
\hline [ALP] & $212.25 \pm 41.05$ & $235.75 \pm 68.86$ & $186.8 \pm 33.89$ \\
\hline [GGT] & $4.67 \pm 1.15$ & $4.01 \pm 0.01$ & $4.22 \pm 1.41$ \\
\hline [GLU] & $8.6 \pm 2.27$ & $8.53 \pm 3.87$ & $11.05 \pm 4.03$ \\
\hline [BUN] & $8.93 \pm 2.4$ & $10.1 \pm 1.42$ & $12.2 \pm 1.83$ \\
\hline [CREA] & $19.33 \pm 1.15$ & $19.5 \pm 1.29$ & $20.75 \pm 0.96$ \\
\hline [UA] & $204.67 \pm 52.6$ & $187.33 \pm 23.97$ & $169.5 \pm 24.12$ \\
\hline [Ca] & $2.44 \pm 0.17$ & $2.4 \pm 0.12$ & $2.39 \pm 0.11$ \\
\hline$[P]$ & $2.72 \pm 0.65$ & $2.9 \pm 0.34$ & $2.5 \pm 0.37$ \\
\hline [CHOI] & $2.58 \pm 0.17$ & $2.48 \pm 0.19$ & $2.85 \pm 0.48$ \\
\hline [TG] & $1.25 \pm 0.37$ & $1.17 \pm 0.32$ & $0.63 \pm 0.12^{\star}$ \\
\hline [HDLC] & $1.48 \pm 0.23$ & $1.65 \pm 0.18$ & $1.69 \pm 0.24$ \\
\hline [LDLC] & $0.46 \pm 0.04$ & $0.48 \pm 0.04$ & $0.44 \pm 0.07$ \\
\hline [LDH] & $1777.25 \pm 519.45$ & $2010.25 \pm 308.79$ & $1344.83 \pm 490.88$ \\
\hline [CKMB] & $437 \pm 127.14$ & $513.25 \pm 26.99$ & $296.17 \pm 48.52^{\star}$ \\
\hline [SOD] & $51 \pm 18.19$ & $70.5 \pm 14.62$ & $92.17 \pm 15.3^{\star \star}$ \\
\hline [CK] & $845.5 \pm 135$ & $948 \pm 163.81$ & $478.2 \pm 82.87^{\star \star \star}$ \\
\hline \multicolumn{4}{|c|}{ Data were presented as mean \pm standard deviation $(n=4)$} \\
\hline \multicolumn{4}{|c|}{ 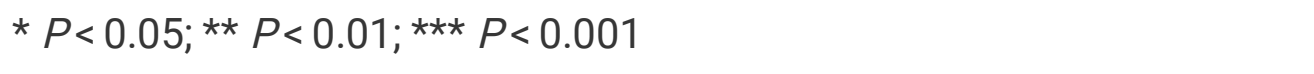 } \\
\hline
\end{tabular}

Since HER2 is a member of the EGFR family, we compared the protein amino acid sequences of four members of the EGFR family (HER1 HER4). It is found that K716 is a homologous amino acid and 
presents in four members (Additional files 8: Figure. S7a). To confirm this, we determined expressions of HER1, HER3 and HER4 in JAC1-treated SKBR3 cells. As predicted, JAC1 dose-dependently suppressed expressions of all EGFR members (Additional files 8: Figure. S7b).

\section{Discussion}

The BC patients usually have favorable prognosis and the average five-year survival is up to $90 \%$. However, the patients with HER2 overexpression, especially those with cancer metastasis, usually have a poor prognosis[25-27]. Herein, we identified for the first time that JAC1, as a specific agonist of JWA gene, could enhance ubiquitin modification of HER2 at K716 by SMURF1 E3 ligase and accelerate its degradation, thereby inhibiting proliferation of BC cells. JAC1 increased the expression of SMURF1 by reducing the expression of NEDD4, thus degrading HER2. In addition, the regulatory effect of JAC1 on NEDD4 relied on the inhibition of the negative transcriptional regulation of GATA-1 through the p38 MAPK pathway. JAC1 suppressed the overexpressed HER2 in BC cells to almost a baseline level and thus maintained the physiological role of HER2 in these cells. Importantly, JAC1 exerted a protective role on liver, myocardial cells, lipid metabolism and anti-oxidant capacity in BC xenografted mice. It is suggested that JAC1 has a potential in drug development and may be utilized as a fundamental therapy to HER2positive BC.

Our previous studies have reported that JWA gene is not only conserved in evolution but has several essential functions, including regulations on cell differentiation, DNA repair, cytoskeleton organization, and metabolisms of sugar and lipid[17, 28, 29]. The biological functions of the JWA gene is achieved largely by regulating the MAPK signaling pathway, which is essential for maintaining basic life activities in organisms[30].

HER2 is known as a member of EGFR family, which contains four members, including EGFR (HER1), HER2 (ErbB2), HER3 and HER4[31]. HER2-positive BC cells are usually contain homo- or heterodimers of EGFR members, such as HER2/HER2 or HER2/HER3[32, 33]. In the present study, we also identified that K716 is a homologue amino acid in all four EGFR family members, and JAC1 could also down-regulate expressions of HER1, HER3 and HER4 in BC cells. The underlying mechanisms and translational significances, however, need to be determined.

The current HER2-targeted therapies (trastuzumab, pertuzumab and lapatinib) have been approved for clinical treatment of HER2-positive BC and largely improved prognosis of HER2-positive cases[34]. Unfortunately, the unavoidable side effects induced by the therapies and drug resistance are always accompanied during the therapeutic period and have become the biggest challenge in clinical application[35,36]. Although the antibody-drug conjugates (ADC) drugs have been identified to display more powerful therapeutic effects than HER2-target antibody alone[37, 38], its side effects are also need to be concerned. In addition, all above drugs or therapies are unable to degrade overexpressed HER2 or HER3, which may inevitably contribute to the development of drug resistance and side effects. The urgent 
need for HER2-positive BC is to develop novel drugs or therapies to degrade the overexpressed HER2 in cancer cells, thereby reversing HER2-induced excessive proliferation and metastasis in BC cells.

In this study, we developed a small molecule compound JAC1 that degraded overexpressed HER2 even in all EGFR members. This will provide new insights into EGFR-overexpression cancer therapies. EGFR family mutations or rearrangements lead to drug resistance, which is the current challenge for targeted drugs. Further studies should to determine if JAC1 is also effective on BC cells with EGFR family mutations or rearrangements.

Previous reports have demonstrated that NEDD4 is associated with BC progression and predicts a poor prognosis. Up-regulation of NEDD4 mediates cell migration in lung cancer cells. Down-regulation of NEDD4 inhibits cell growth and invasion, and induces cell apoptosis in bladder cancer cells[39-41]. These evidences supported the anticancer function of JAC1 as an indirect inhibitor on NEDD4.

\section{Conclusion}

In summary, JAC1, an agonist of JWA gene, was identified as an indirect inhibitor of HER2. JAC1 degrades overexpressed HER2 in BC by ubiquitination mechanism through the p38 MAPK signaling pathway. JAC1 may have important translational significance in cancer therapy.

\section{Abbreviations}

$\mathrm{BC}$

Breast cancer; CHX:Cycloheximide; Co-IP:Co-immunoprecipitation; DMSO:Dimethyl sulfoxide; OS:overall survival; PBS:Phosphate buffered solution; SMURF1:SMAD specific E3 ubiquitin protein ligase 1; TBS:Tris-buffered saline; TMA:Tissue microarrays; Ub:Ubiquitin.

\section{Declarations}

\section{Ethics approval and consent to participate}

The study was approved by the Ethics Committee of Nanjing Medical University.

\section{Consent for publication}

Consent was achieved from all patients.

\section{Availability of data and materials}

All other data are available in the main text or the supplementary materials. The datasets used and/or analyzed during the current study are available from the websites mentioned in the text.

\section{Competing interests}


The authors declare that they have no competing interests.

\section{Funding}

This work was supported by the National Natural Science Foundation of China (Nos. 81520108027 , $81673219,81521004,81973156$ and 30930080).

\section{Authors' Contributions}

Conception and design: Y. Ren, J. Zhou

Development of methodology: Y. Ren, J. Chen, Z. Zhai, D. Chen, A. Li, Y. Liang

Acquisition of data: Y. Ren, J. Chen, Z. Zhai, D. Chen, Y. Liang

Analysis and interpretation of data: Y. Ren, J. Chen, Z. Zhai, D. Chen,

Writing, review, and/or revision of the manuscript: Y. Ren, J. Chen and J. Zhou

Administrative, technical, or material support: Y. Ren, J. Chen, Z. Zhai, D. Chen, A. Li

Study supervision: J. Zhou

\section{Acknowledgements}

We thank Ms. Jianping Xiong from Nanjing Medical University for her great support in using confocal microscope.

\section{References}

1. Bray F, Ferlay J, Soerjomataram I, Siegel RL, Torre LA, Jemal A: Global cancer statistics 2018: GLOBOCAN estimates of incidence and mortality worldwide for 36 cancers in 185 countries. CA: a cancer journal for clinicians 2018, 68(6):394-424.

2. DeSantis C, Howlader N, Cronin KA, Jemal A: Breast cancer incidence rates in U.S. women are no longer declining. Cancer epidemiology, biomarkers \& prevention : a publication of the American Association for Cancer Research, cosponsored by the American Society of Preventive Oncology 2011, 20(5):733-739.

3. DeSantis C, Ma J, Bryan L, Jemal A: Breast cancer statistics, 2013. CA: a cancer journal for clinicians 2014, 64(1):52-62.

4. Cancer Genome Atlas N: Comprehensive molecular portraits of human breast tumours. Nature 2012, 490(7418):61-70.

5. Moasser MM: Targeting the function of the HER2 oncogene in human cancer therapeutics. Oncogene 2007, 26(46):6577-6592. 
6. Romond EH, Perez EA, Bryant J, Suman VJ, Geyer CE, Jr., Davidson NE, Tan-Chiu E, Martino S, Paik S, Kaufman PA et al: Trastuzumab plus adjuvant chemotherapy for operable HER2-positive breast cancer. The New England journal of medicine 2005, 353(16):1673-1684.

7. Scheuer W, Friess T, Burtscher H, Bossenmaier B, Endl J, Hasmann M: Strongly enhanced antitumor activity of trastuzumab and pertuzumab combination treatment on HER2-positive human xenograft tumor models. Cancer research 2009, 69(24):9330-9336.

8. Geyer CE, Forster J, Lindquist D, Chan S, Romieu CG, Pienkowski T, Jagiello-Gruszfeld A, Crown J, Chan A, Kaufman B et al: Lapatinib plus capecitabine for HER2-positive advanced breast cancer. The New England journal of medicine 2006, 355(26):2733-2743.

9. Wardley AM, Pivot X, Morales-Vasquez F, Zetina LM, de Fatima Dias Gaui M, Reyes DO, Jassem J, Barton $\mathrm{C}$, Button $\mathrm{P}$, Hersberger $\mathrm{V}$ et al: Randomized phase II trial of first-line trastuzumab plus docetaxel and capecitabine compared with trastuzumab plus docetaxel in HER2-positive metastatic breast cancer. Journal of clinical oncology : official journal of the American Society of Clinical Oncology 2010, 28(6):976-983.

10. Reid A, Vidal L, Shaw H, de Bono J: Dual inhibition of ErbB1 (EGFR/HER1) and ErbB2 (HER2/neu). European journal of cancer 2007, 43(3):481-489.

11. Giordano SH, Temin S, Kirshner JJ, Chandarlapaty S, Crews JR, Davidson NE, Esteva FJ, GonzalezAngulo AM, Krop I, Levinson J et al: Systemic therapy for patients with advanced human epidermal growth factor receptor 2-positive breast cancer: American Society of Clinical Oncology clinical practice guideline. Journal of clinical oncology : official journal of the American Society of Clinical Oncology 2014, 32(19):2078-2099.

12. Wang S, Wu X, Chen Y, Zhang J, Ding J, Zhou Y, He S, Tan Y, Qiang F, Bai J et al: Prognostic and predictive role of JWA and XRCC1 expressions in gastric cancer. Clinical cancer research : an official journal of the American Association for Cancer Research 2012, 18(10):2987-2996.

13. Bai J, Zhang J, Wu J, Shen L, Zeng J, Ding J, Wu Y, Gong Z, Li A, Xu S et al: JWA regulates melanoma metastasis by integrin alphaVbeta3 signaling. Oncogene 2010, 29(8):1227-1237.

14. Xu L, Cheng L, Yang F, Pei B, Liu X, Zhou J, Zhu Y, Wang S: JWA suppresses the invasion of human breast carcinoma cells by downregulating the expression of CXCR4. Molecular medicine reports 2018, 17(6):8137-8144.

15. Lu J, Tang Y, Farshidpour M, Cheng Y, Zhang G, Jafarnejad SM, Yip A, Martinka M, Dong Z, Zhou J et al: JWA inhibits melanoma angiogenesis by suppressing ILK signaling and is an independent prognostic biomarker for melanoma. Carcinogenesis 2013, 34(12):2778-2788.

16. Wu X, Chen H, Gao Q, Bai J, Wang X, Zhou J, Qiu S, Xu Y, Shi Y, Wang X et al: Downregulation of JWA promotes tumor invasion and predicts poor prognosis in human hepatocellular carcinoma. Molecular carcinogenesis 2014, 53(4):325-336.

17. Qian J, Zhu W, Wang K, Ma L, Xu J, Xu T, Roe OD, Li A, Zhou J, Shu Y: JWA loss promotes cell migration and cytoskeletal rearrangement by affecting HER2 expression and identifies a high-risk subgroup of HER2-positive gastric carcinoma patients. Oncotarget 2016, 7(24):36865-36884. 
18. Ma L, Zhu W, Wang Q, Yang F, Qian J, Xu T, Wang S, Zhou J, Shu Y: JWA down-regulates HER2 expression via c-Cbl and induces lapatinib resistance in human gastric cancer cells. Oncotarget 2016, 7(44):71790-71801.

19. Zhou J, Ye J, Zhao X, Li A, Zhou J: JWA is required for arsenic trioxide induced apoptosis in HeLa and MCF-7 cells via reactive oxygen species and mitochondria linked signal pathway. Toxicology and applied pharmacology 2008, 230(1):33-40.

20. Ran FA, Hsu PD, Wright J, Agarwala V, Scott DA, Zhang F: Genome engineering using the CRISPRCas9 system. Nature protocols 2013, 8(11):2281-2308.

21. Qiu D, Wang Q, Wang Z, Chen J, Yan D, Zhou Y, Li A, Zhang R, Wang S, Zhou J: RNF185 modulates JWA ubiquitination and promotes gastric cancer metastasis. Biochimica et biophysica acta Molecular basis of disease 2018, 1864(5 Pt A):1552-1561.

22. Li Y, Xie P, Lu L, Wang J, Diao L, Liu Z, Guo F, He Y, Liu Y, Huang Q et al: An integrated bioinformatics platform for investigating the human E3 ubiquitin ligase-substrate interaction network. Nature communications 2017, 8(1):347.

23. Chen H, Bai J, Ye J, Liu Z, Chen R, Mao W, Li A, Zhou J: JWA as a functional molecule to regulate cancer cells migration via MAPK cascades and F-actin cytoskeleton. Cellular signalling 2007, 19(6):1315-1327.

24. Bibikova E, Youn MY, Danilova N, Ono-Uruga Y, Konto-Ghiorghi Y, Ochoa R, Narla A, Glader B, Lin S, Sakamoto KM: TNF-mediated inflammation represses GATA1 and activates p38 MAP kinase in RPS19-deficient hematopoietic progenitors. Blood 2014, 124(25):3791-3798.

25. Santa-Maria CA, Nye L, Mutonga MB, Jain S, Gradishar WJ: Management of Metastatic HER2Positive Breast Cancer: Where Are We and Where Do We Go From Here? Oncology 2016, 30(2):148155.

26. Chen CY, Yang CY, Chen YC, Shih CW, Lo SS, Lin CH: Decreased expression of stomatin predicts poor prognosis in HER2-positive breast cancer. BMC cancer 2016, 16:697.

27. Slamon DJ, Clark GM, Wong SG, Levin WJ, Ullrich A, McGuire WL: Human breast cancer: correlation of relapse and survival with amplification of the HER-2/neu oncogene. Science 1987, 235(4785):177182.

28. Chen R, Qiu W, Liu Z, Cao X, Zhu T, Li A, Wei Q, Zhou J: Identification of JWA as a novel functional gene responsive to environmental oxidative stress induced by benzo[a]pyrene and hydrogen peroxide. Free radical biology \& medicine 2007, 42(11):1704-1714.

29. Wang S, Gong Z, Chen R, Liu Y, Li A, Li G, Zhou J: JWA regulates XRCC1 and functions as a novel base excision repair protein in oxidative-stress-induced DNA single-strand breaks. Nucleic acids research 2009, 37(6):1936-1950.

30. Sun Y, Liu WZ, Liu T, Feng X, Yang N, Zhou HF: Signaling pathway of MAPK/ERK in cell proliferation, differentiation, migration, senescence and apoptosis. Journal of receptor and signal transduction research 2015, 35(6):600-604. 
31. Yarden Y: The EGFR family and its ligands in human cancer. signalling mechanisms and therapeutic opportunities. European journal of cancer 2001, 37 Suppl 4:S3-8.

32. Hendriks BS, Opresko LK, Wiley HS, Lauffenburger D: Quantitative analysis of HER2-mediated effects on HER2 and epidermal growth factor receptor endocytosis: distribution of homo- and heterodimers depends on relative HER2 levels. The Journal of biological chemistry 2003, 278(26):23343-23351.

33. Yarden Y, Sliwkowski MX: Untangling the ErbB signalling network. Nature reviews Molecular cell biology 2001, 2(2):127-137.

34. Murthy RK, Loi S, Okines A, Paplomata E, Hamilton E, Hurvitz SA, Lin NU, Borges V, Abramson V, Anders $C$ et al: Tucatinib, Trastuzumab, and Capecitabine for HER2-Positive Metastatic Breast Cancer. The New England journal of medicine 2020, 382(7):597-609.

35. Chun KH, Park JH, Fan S: Predicting and Overcoming Chemotherapeutic Resistance in Breast Cancer. Advances in experimental medicine and biology 2017, 1026:59-104.

36. Vernieri C, Milano M, Brambilla M, Mennitto A, Maggi C, Cona MS, Prisciandaro M, Fabbroni C, Celio L, Mariani G et al: Resistance mechanisms to anti-HER2 therapies in HER2-positive breast cancer: Current knowledge, new research directions and therapeutic perspectives. Critical reviews in oncology/hematology 2019, 139:53-66.

37. Thomas A, Teicher BA, Hassan R: Antibody-drug conjugates for cancer therapy. The Lancet Oncology 2016, 17(6):e254-e262.

38. Beck A, Goetsch L, Dumontet C, Corvaia N: Strategies and challenges for the next generation of antibody-drug conjugates. Nature reviews Drug discovery 2017, 16(5):315-337.

39. Shao G, Wang R, Sun A, Wei J, Peng K, Dai Q, Yang W, Lin Q: The E3 ubiquitin ligase NEDD4 mediates cell migration signaling of EGFR in lung cancer cells. Molecular cancer 2018, 17(1):24.

40. Wen W, Li J, Wang L, Xing Y, Li X, Ruan H, Xi X, Xiong J, Kuang R: Inhibition of NEDD4 inhibits cell growth and invasion and induces cell apoptosis in bladder cancer cells. Cell cycle 2017, 16(16):15091514.

41. Wan L, Liu T, Hong Z, Pan Y, Sizemore ST, Zhang J, Ma Z: NEDD4 expression is associated with breast cancer progression and is predictive of a poor prognosis. Breast cancer research : BCR 2019, 21(1):148.

\section{Figures}


Figure 1

a
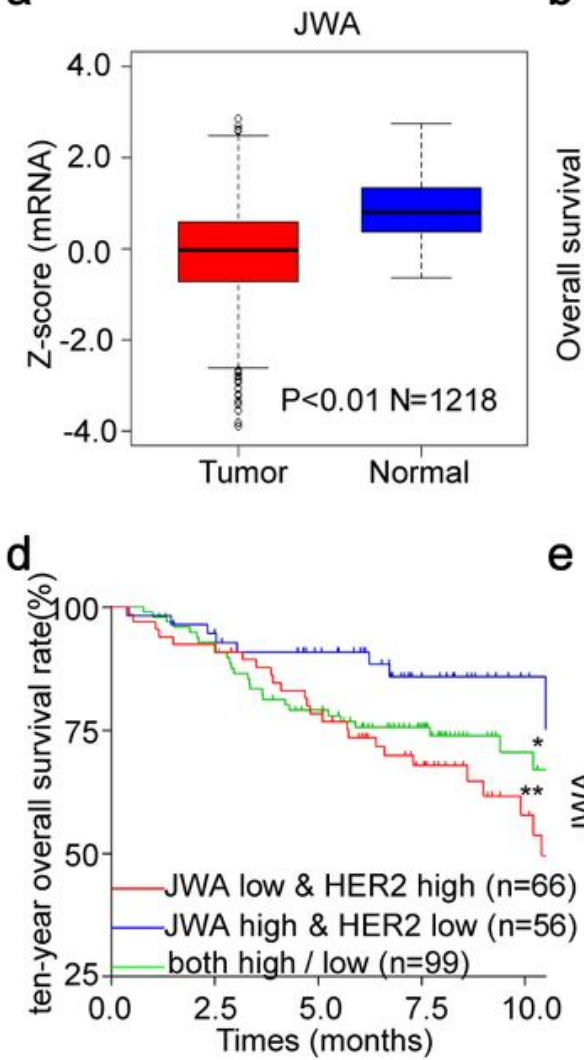

g

BT474

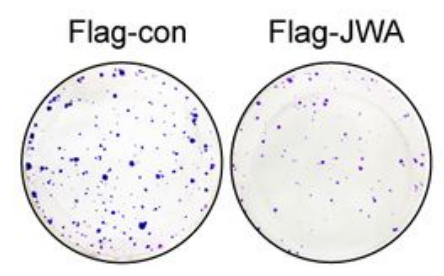

h

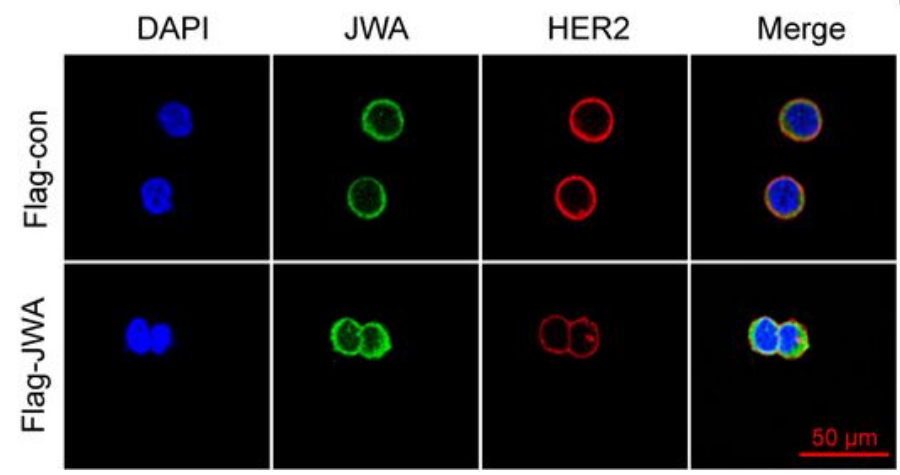

b

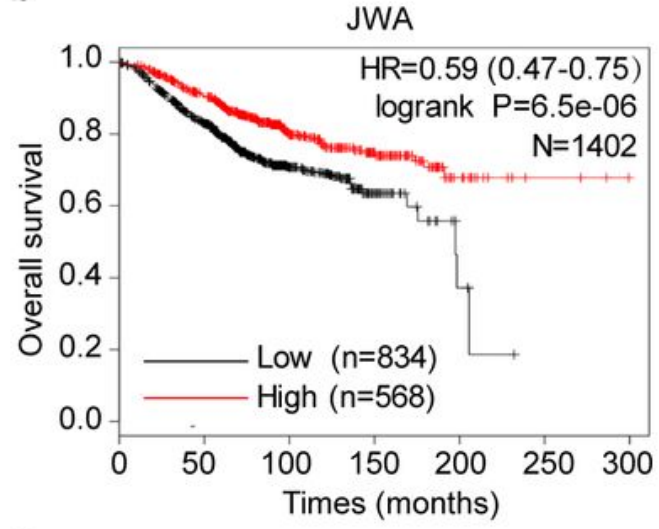

e

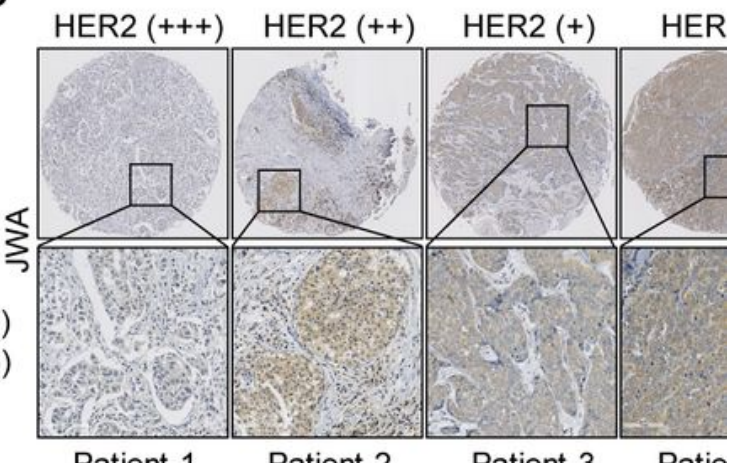

Patient 1 Patient $2 \quad$ Patient $3 \quad$ Patie

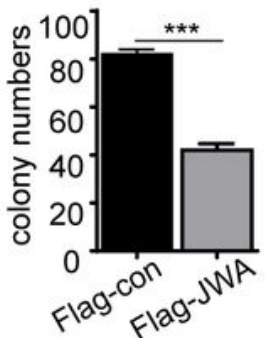

BT474

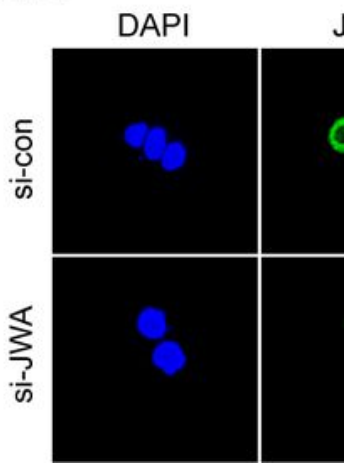

\section{Figure 1}

JWA negatively regulates HER2 expression and cell proliferation in BC. a The mRNA expressions of JWA in $B C$ and adjacent noncancerous tissues in unpaired cohorts (TCGA database, 1099 cancer samples, 119 noncancerous samples). b-c Kaplan-Meier curves depicting OS according to the expression patterns of JWA (b) and HER2 (c) in the BC cohort. P values were calculated with the log-rank test. $d$ Kaplan-Meier OS analysis for BC from GEO datasets (GSE88770 and GES42568). e Representative images of JWA IHC 
staining in BC lesions with different HER2 scores (original magnification, $\times 2$ and $\times 200$ ). $f$ Transfection of either Flag-JWA plasmid or si-JWA and their control into BT474 cells for $72 \mathrm{~h}$, the levels of HER2 and JWA proteins were determined by western blot. $g$ Representative images of the colony formation assay for BT474 after transfection of Flag-JWA or si-JWA and their control cells. h Immunofluorescence imaging of JWA (green), HER2 (red), nucleus labeled as DAPI (blue), the co-localization of the three signals (merge) in BT474 cells transfected with si-JWA (right) or Flag-JWA for $72 \mathrm{~h}$. BC, breast cancer; OS, overall survival.

Figure 2

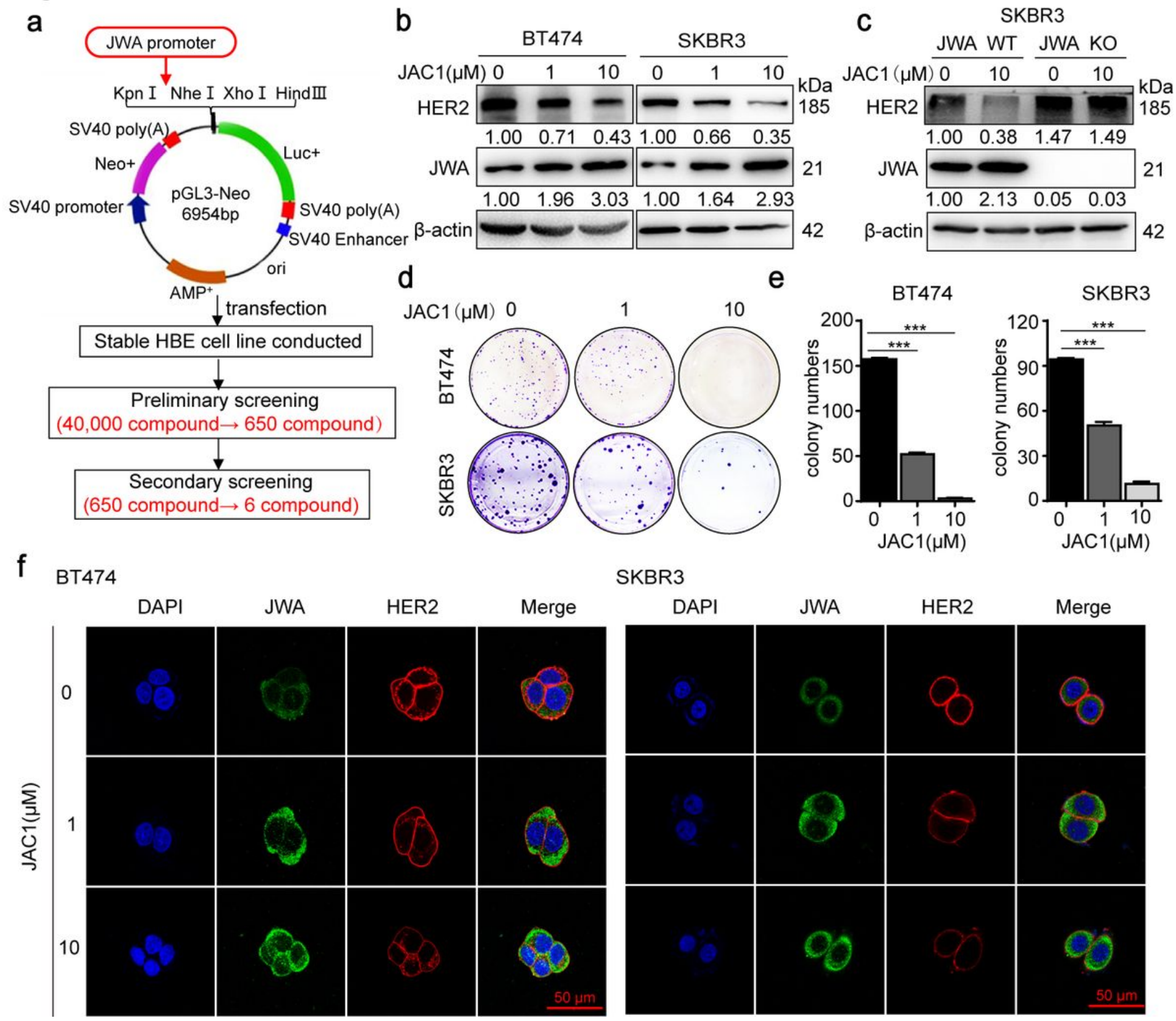

Figure 2

JAC1 down-regulates HER2 expression and inhibits cell proliferation in BC. a Flow chart of screening strategy for small molecule compounds JWA gene agonist. b Protein levels of the HER2 and JWA were determined by Western blot. c JWA/wild type or JWA/KO cells were treated with JAC1 (10 $\mu \mathrm{M})$ for $24 \mathrm{~h}$. The HER2 and JWA protein levels were determined by Western blot. d-e Representative images of the 
colony formation assay for BT474 and SKBR3 cells after treatment with different concentrations of JAC1 $(0,1,10 \mu \mathrm{M})$. f BT474 and SKBR3 cells were treated with JAC1 $(0,1,10 \mu \mathrm{M})$ for $24 \mathrm{~h}$, immunofluorescence imaging showed expressions of JWA (green), HER2 (red), nucleus (blue), the colocalization of the three signals (merge).

\section{Figure 3}

a

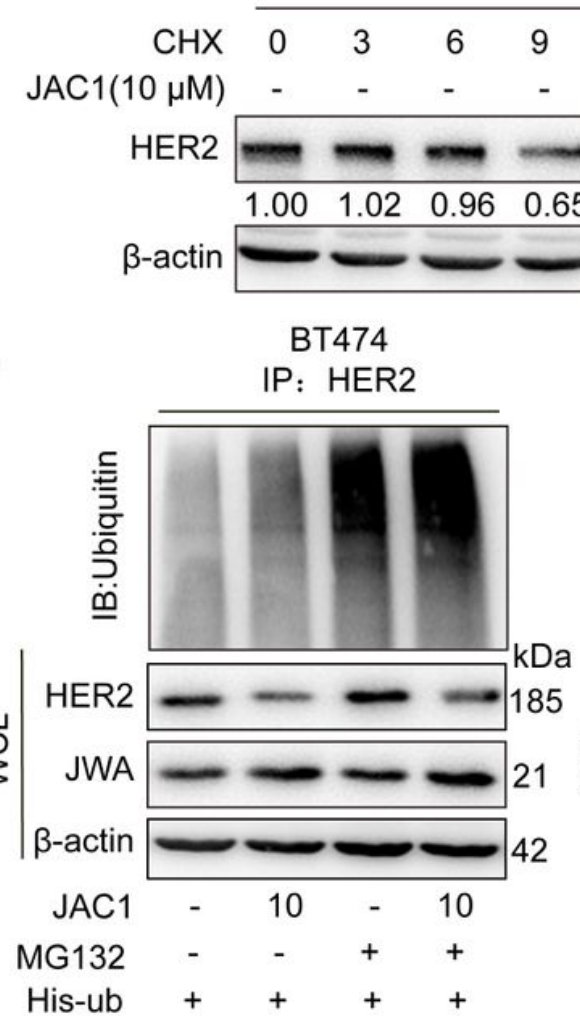

e

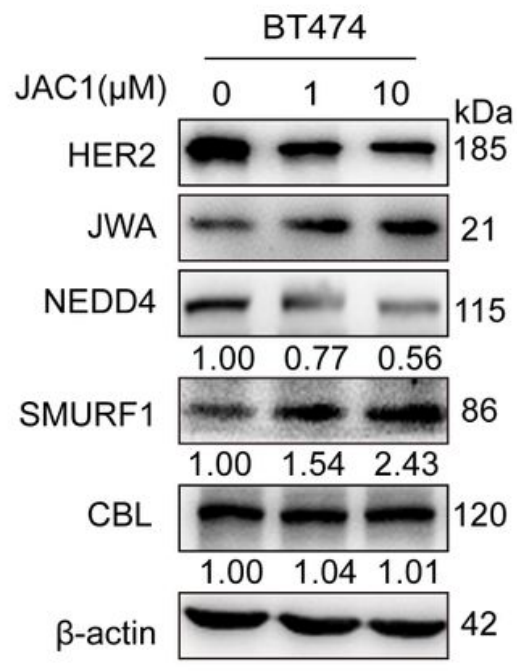

BT474

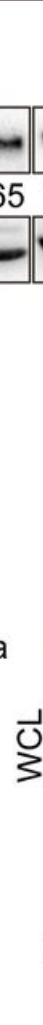

$\begin{array}{llll}0 & 3 & 6 & 9 \mathrm{~h}\end{array}$

$++++k \mathrm{kDa}$

$--\longrightarrow 185$

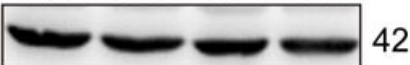

C
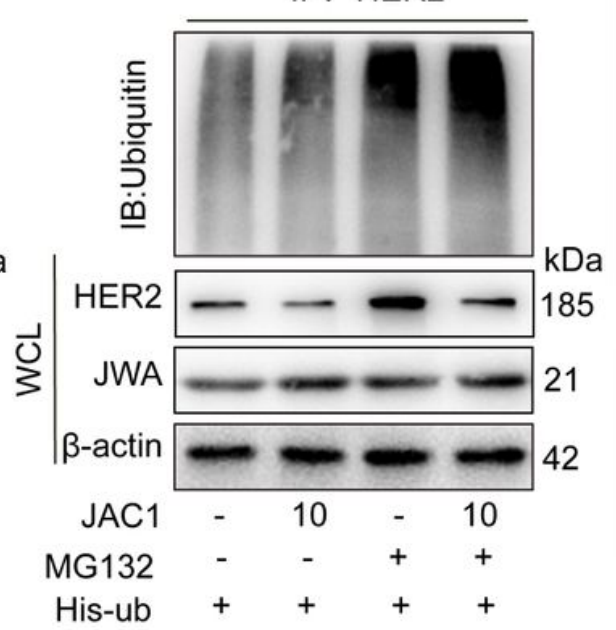

d

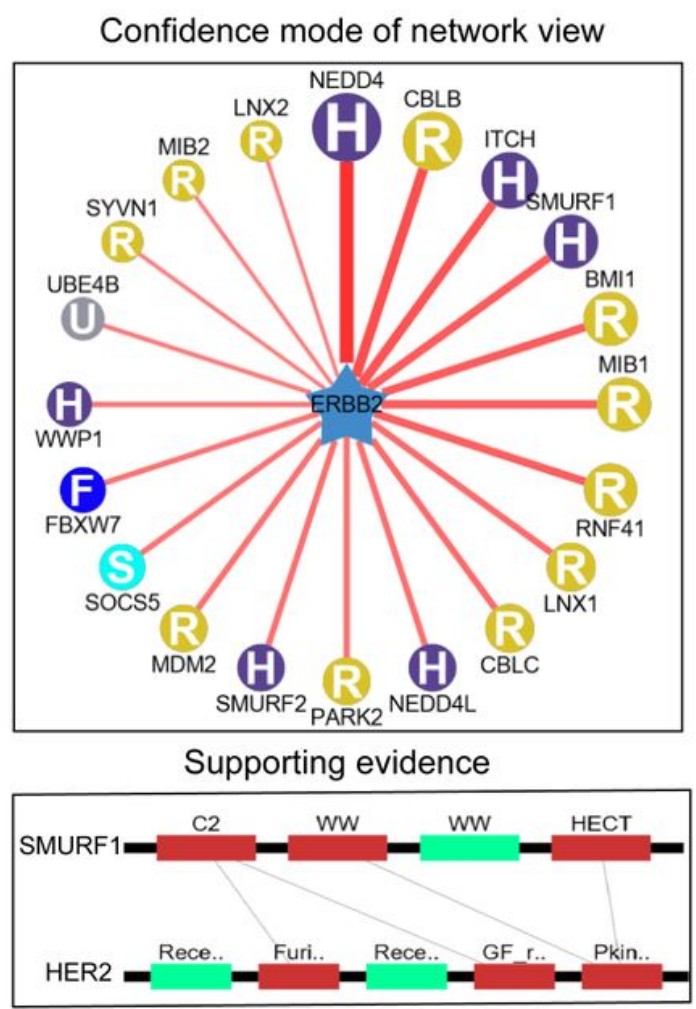

$\mathrm{f}$
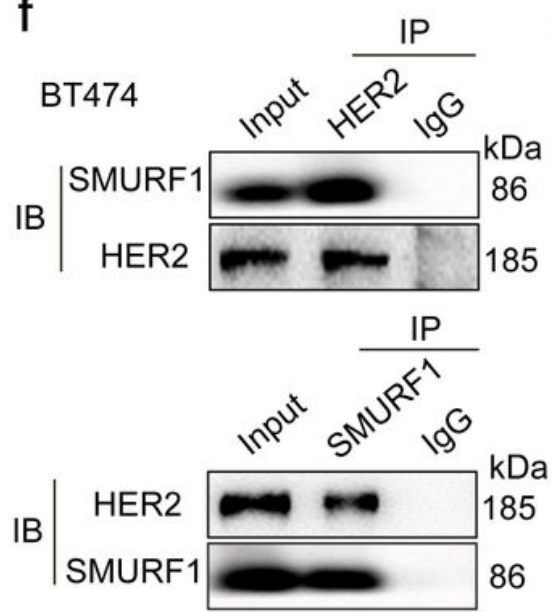

g

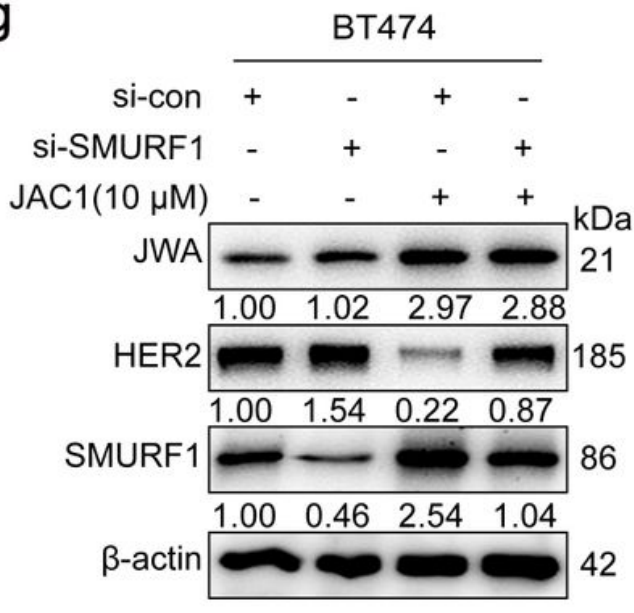

\section{Figure 3}

JAC1 down-regulates HER2 expression via ubiquitin-proteasome pathway. a BT474 cells were treated with the JAC1 $(0,1,10 \mu \mathrm{M})$ for $24 \mathrm{~h}$, followed by CHX $(100 \mu \mathrm{g} / \mathrm{ml})$ for $0,3,6,9 \mathrm{~h}$. HER2 protein levels were determined by western blot. b-c Ubiquitination of HER2 was induced by JAC1. Ubiquitination of the HER2 
protein was immunoprecipitated and detected a ubiquitin antibody. $d$ The HER2-targetting E3 ubiquitin ligases were predicted using the public database website (ubibrowser.ncpsb.org/). e The effects of JAC1 on E3 ligase were determined in BT474 cells by Western blot. f BT474 cells were pretreated with MG132 $(10 \mu \mathrm{M})$ for $6 \mathrm{~h}$, and the endogenous protein-protein interaction between SMURF1 and HER2 were assessed by IP and followed by Western blot. $\mathrm{g}$ Western blotting analysis of HER2 and SMURF1 of BT474 cell treated with JAC1 or si-SMURF1 and corresponding controls.

\section{Figure 4}

a

\begin{tabular}{|c|c|c|c|c|c|}
\hline $\mathrm{K} 150$ & <175 & & & \multicolumn{2}{|c|}{ 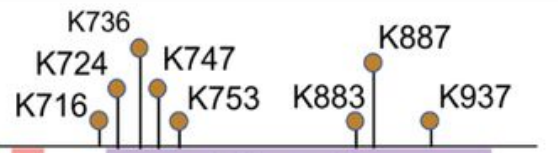 } \\
\hline Recep Ld & Furin-like & Recep Ld & GF recep I & T $\quad$ Pkinase Tyr & \\
\hline 100 & 300 & 400 & 600 & 800 & 1000 \\
\hline
\end{tabular}

b
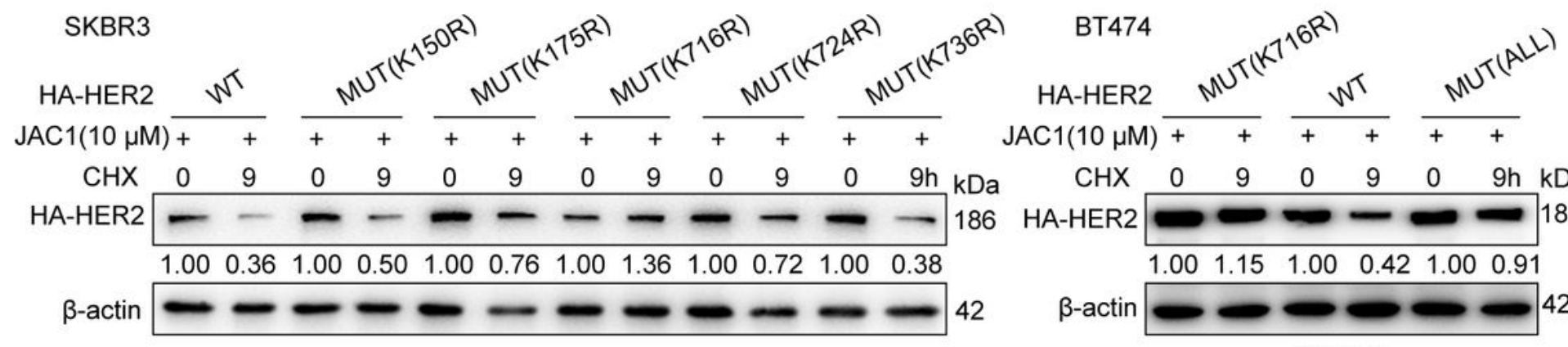

$\mathrm{CHX} \quad 0 \quad \begin{array}{lllllll}0 & 0 & 9 & 0 & 9 \mathrm{~h} & \mathrm{kDa}\end{array}$ HA-HER2

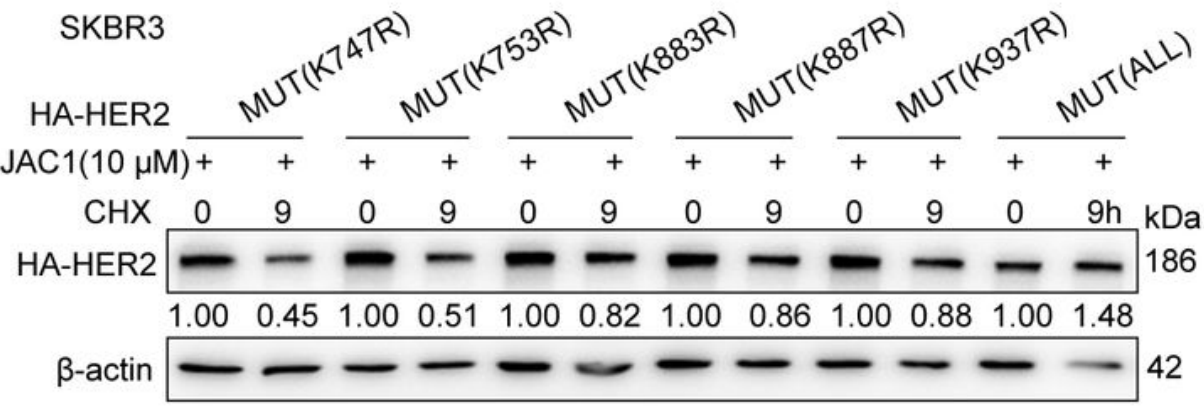

d SKBR3 $\begin{array}{llllll}1.00 & 1.15 & 1.00 & 0.42 & 1.00 & 0.91\end{array}$ $\beta$-actin $40 \longrightarrow 02$ IP: HER2
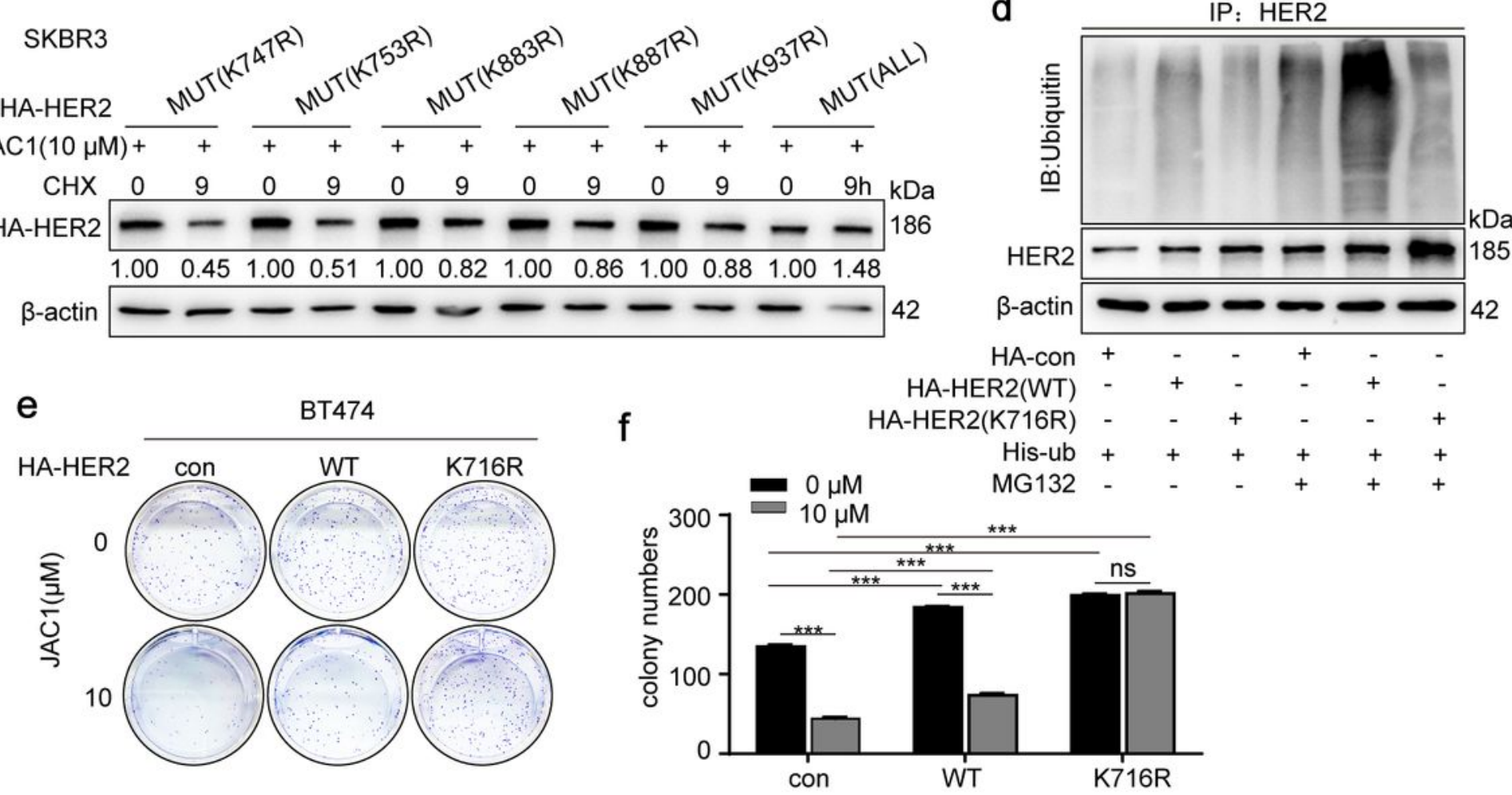

Figure 4 
K716 of HER2 is the ubiquitination target by SMURF1. a The predicted ten potential ubiquitination targets in HER2. b SKBR3 cells were transfected with His-HER2 (WT) or 11 mutants (10 separate single amino acid mutants and all 10 mutated amino acids in HER2), treated with the $10 \mu \mathrm{M} \mathrm{JAC} 1$ for $24 \mathrm{~h}$, followed by exposure to $\mathrm{CHX}(100 \mu \mathrm{g} / \mathrm{ml})$ for $9 \mathrm{~h}$. The HER2 expressions were detected by Western blot. c BT474 cells were transfected with HA-HER2 (WT), HA-HER2 (K716) or HA-HER2 (ALL) for $72 \mathrm{~h}$, treated with the $10 \mu \mathrm{M}$ JAC1 for $24 \mathrm{~h}$, followed by exposure to $100 \mu \mathrm{g} / \mathrm{ml}$ of $\mathrm{CHX}$ for $9 \mathrm{~h}$; the protein level of HER2 was determined by Western blot. d SKBR3 cells were co-transfected with His-Ub, HA-HER2 (WT) or HA-HER2 (K716) for $72 \mathrm{~h}$, followed by pretreatment with MG132 $(10 \mu \mathrm{M})$ for $6 \mathrm{~h}$. Ubiquitinated HA-HER2 was determined by IP followed immunoblot. e Colony formation assay was completed for WT/K716R HER2 transfected BT474 cells and followed by JAC1 $(10 \mu \mathrm{M})$ treatment. $f$ The quantitative data of colony numbers. 


\section{Figure 5}

a

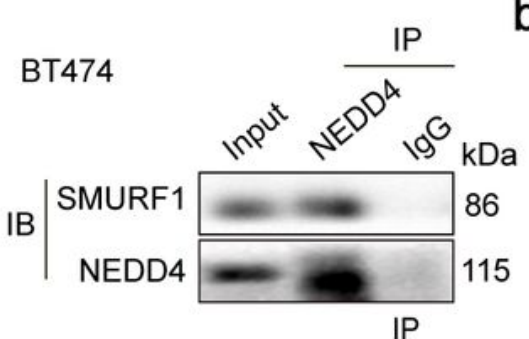

IB

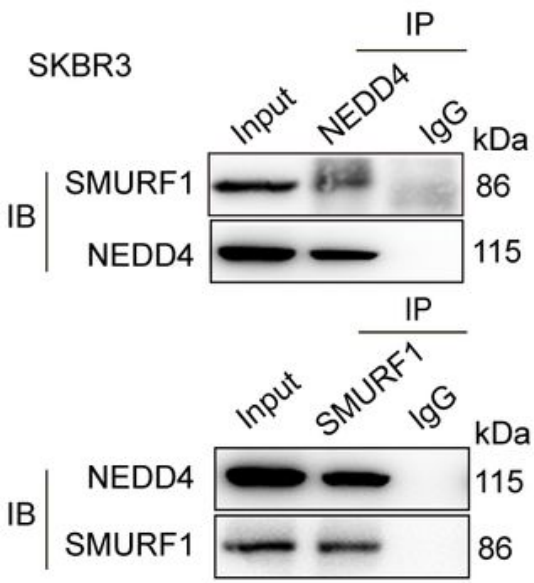

C
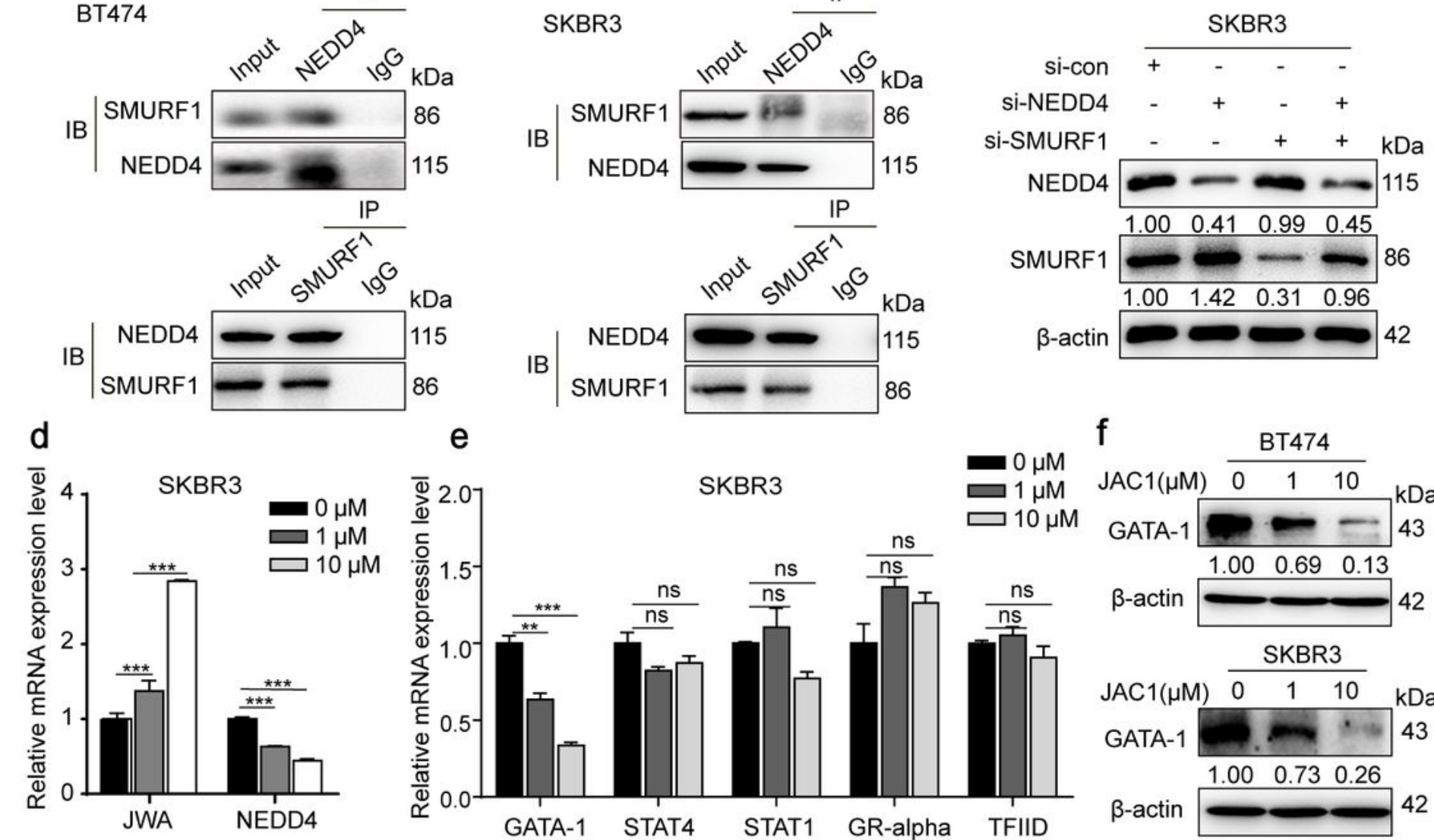

e

g

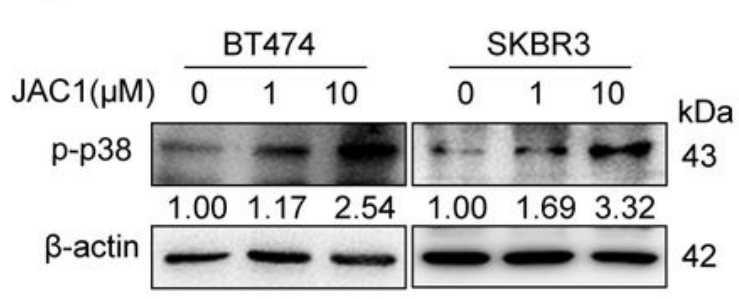

$\mathrm{h}$
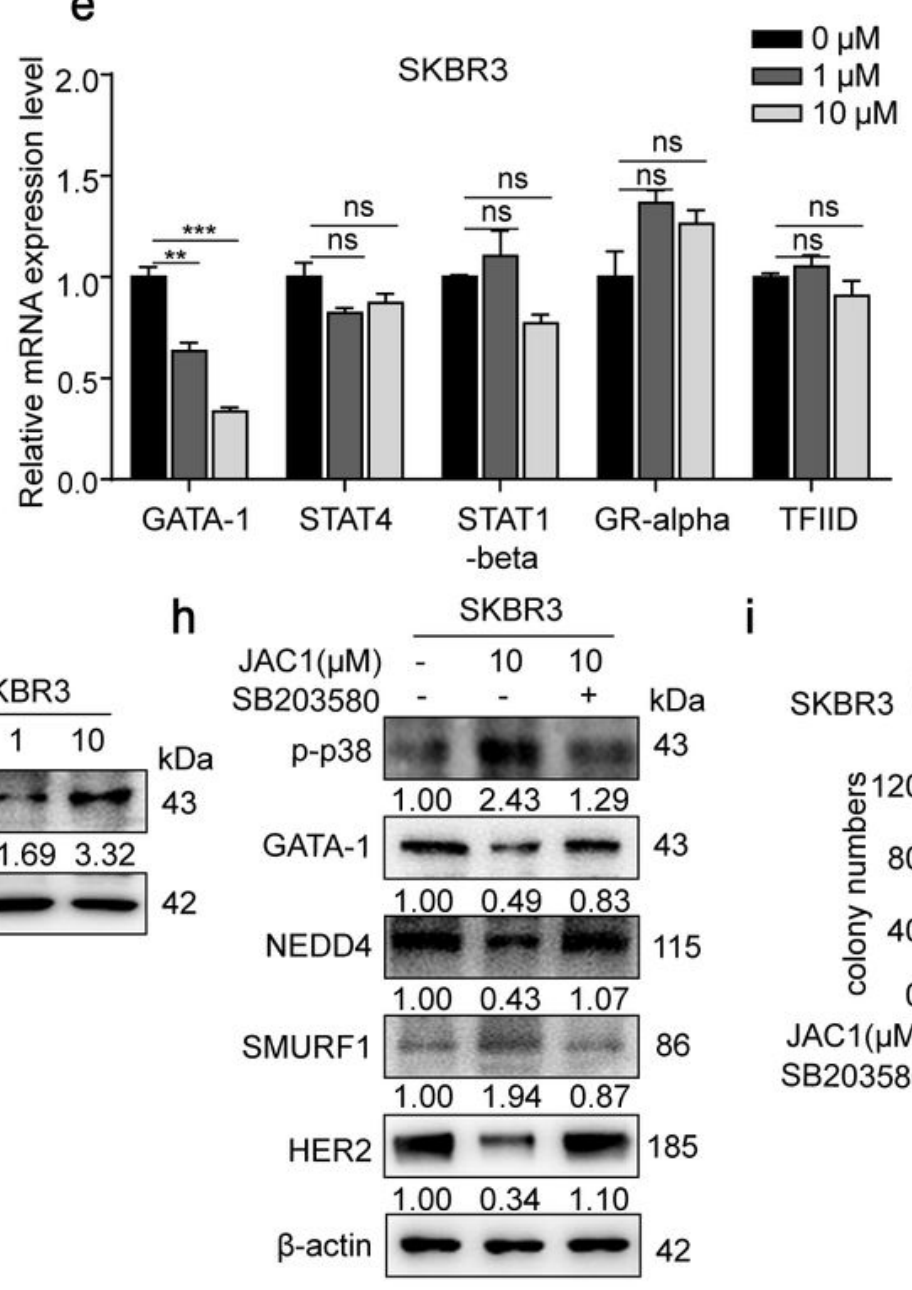
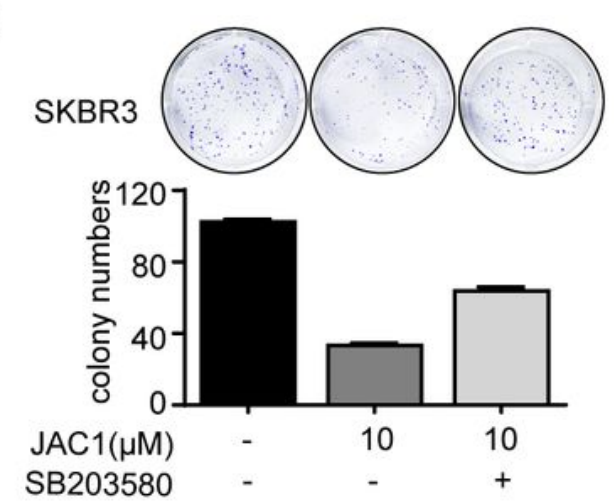

\section{Figure 5}

JAC1 modulates SMURF1 through JWA-p38-GATA-1-NEDD4 axis. a-b The endogenous protein-protein interaction between NEDD4 and SMURF1 were identified by IP and Western blot in both BT474 and SKBR3 cells. c Western blotting analysis of NEDD4 and SMURF1 of SKBR3 cells transfected with siNEDD4 or si-SMURF1. $d$ The mRNA levels of NEDD4 and JWA were detected by real-time PCR in SKBR3. The values were calculated as $\neg 2$-ddCT, and the relative fold change was compared to the control groups 
after being normalized to GAPDH. e The mRNA levels of GATA-1, STAT4, STAT1-beta, GR-alpha and TFIID were detected by real-time PCR in SKBR3. The values were calculated as $\neg 2$-ddCT, and the relative fold change was compared to the control groups after being normalized to GAPDH. $f$ The regulations of JAC1 on GATA-1 was determined in both BT474 and SKBR3 cells. g JAC1-induced activation of p-p38 was determined by Western blot in both BT474 and SKBR3 cells. h Western blotting analysis of p-p38, GATA-1, NEDD4, SMURF1 and HER2 of SKBR3 cells treated with JAC1 or SB203580. i The inhibition of JAC1 on colony formation via the 38 MAK pathway was determined in SKBR3 cells.

\section{Figure 6}

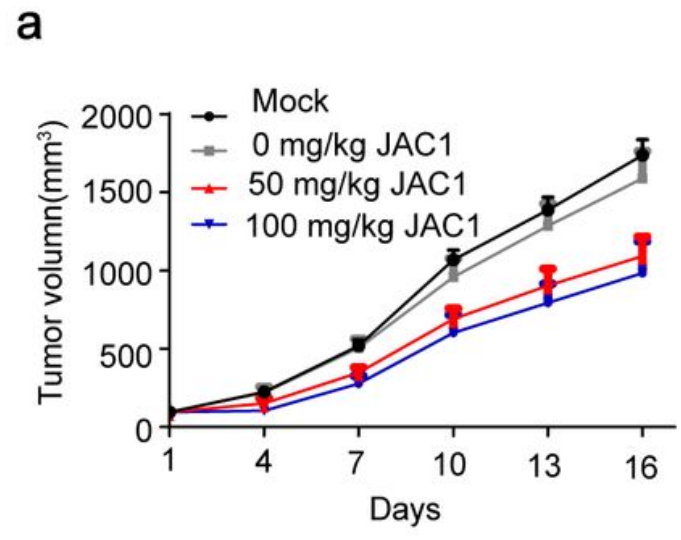

d

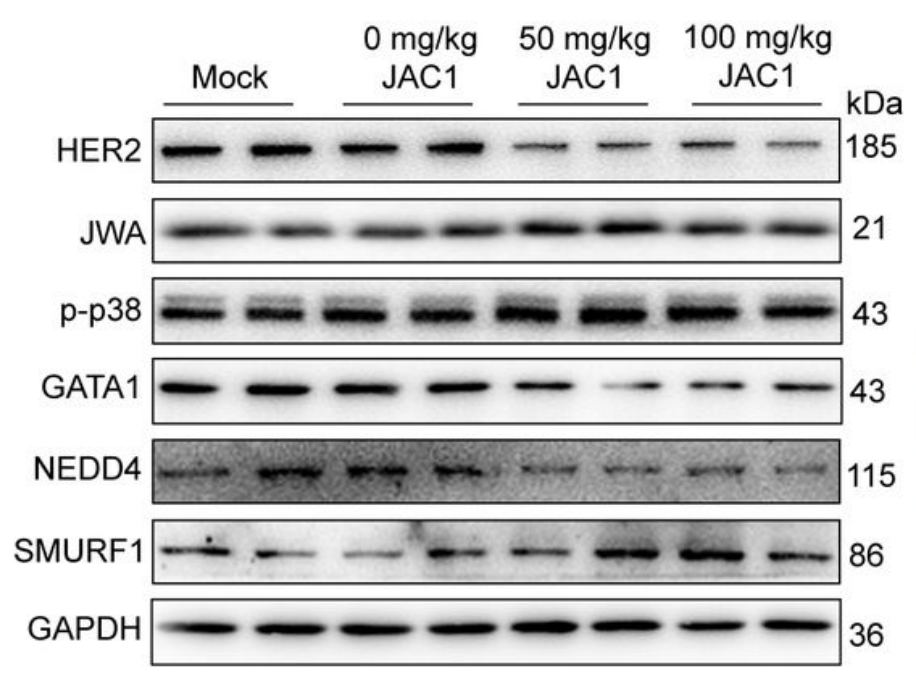

b

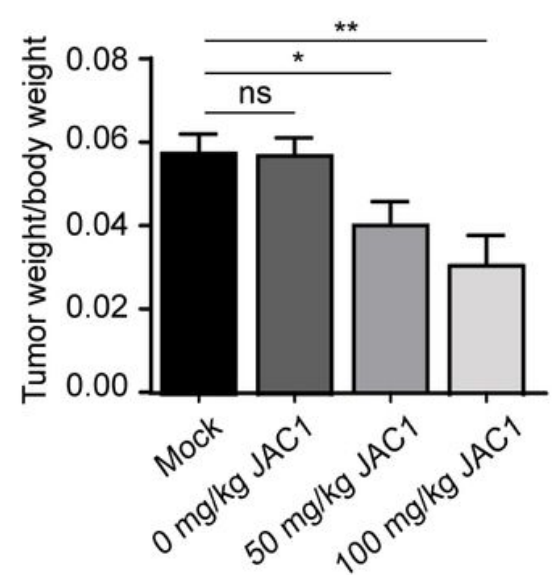

C

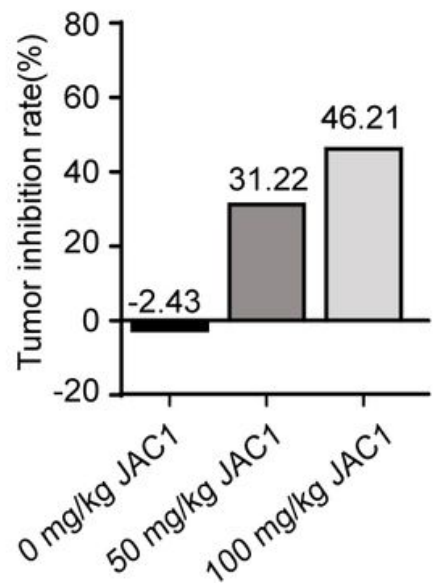

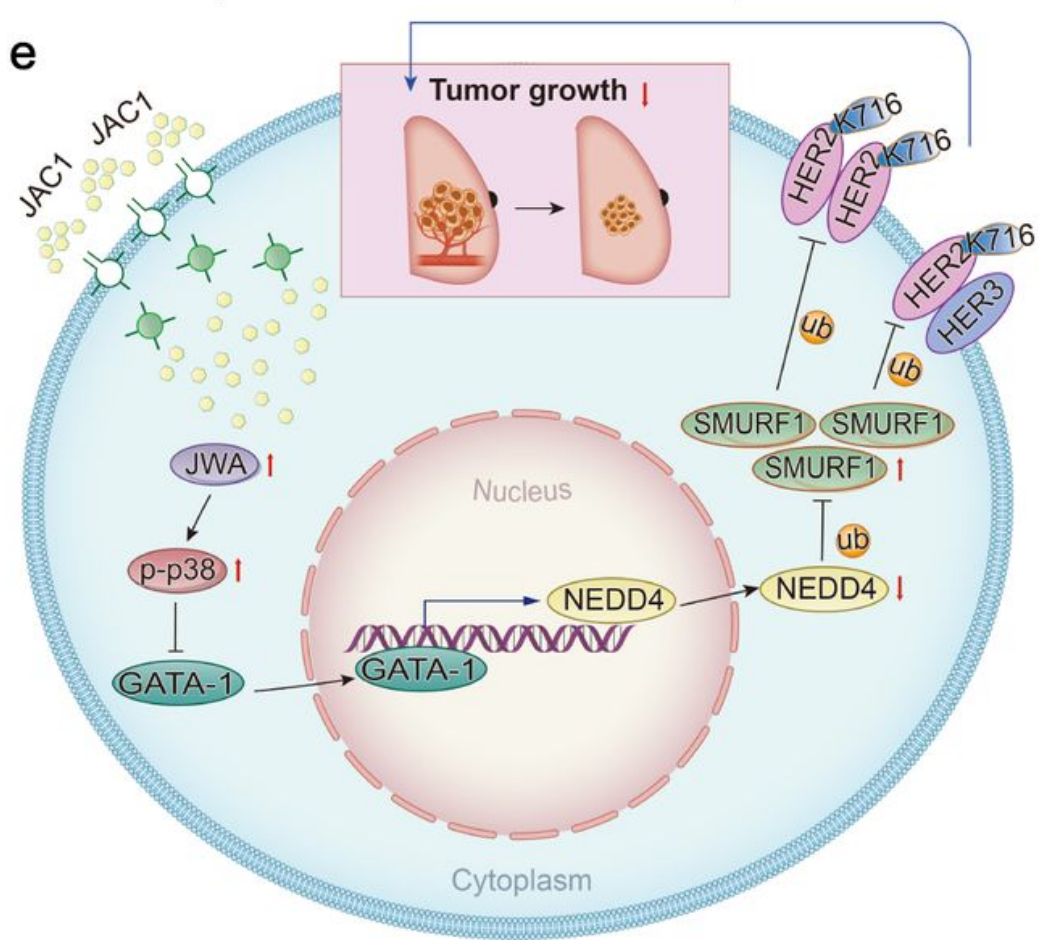

Figure 6

JAC1 modulates HER2 ubiquitination and inhibits tumor growth in vivo. a The curve of tumor growth of BT474 xenografted breast cancer after JAC1 treatment. b-c The ratio of tumor weight/body weight in each group (b), tumor inhibition rate by JAC1 (c). $d$ The expressions of HER2, cell proliferation and mechanistic biomarkers in isolated representative tumor tissues of each group; all the biomarkers were 
determined by Western blot. e A schematic overview how JAC1 degrades HER2 in breast cancer cells. * $P$ $<0.05 ; * \star P<0.01 ; \star \star \star P<0.001$. N.S., no significant differences.

\section{Supplementary Files}

This is a list of supplementary files associated with this preprint. Click to download.

- Fig.S1.tif

- Fig.S2.tif

- Fig.S3.tif

- Fig.S4.tif

- Fig.S5.tif

- Fig.S6.tif

- Fig.S7.tif

- Additionalfiles1.docx 